
\title{
25 Research Soure \\ Effect of Omalizumab for Autoimmune \\ Progesterone Dermatitis Refractory to Bilateral Oophorectomy: a case report
}

\section{Akshay Varghese ( $\square$ avarghese@nosm.ca )}

Northern Ontario School of Medicine at Laurentian University: Northern Ontario School of Medicine East Campus https://orcid.org/0000-0001-9313-0661

\section{Terri Paul}

Western University Schulich School of Medicine and Dentistry

\section{Harold Kim}

Western University Schulich School of Medicine and Dentistry

\section{Stan Van Uum}

Western University Schulich School of Medicine and Dentistry

\section{Peter Vadas}

University of Toronto

\begin{abstract}
Alescia Azzola
Northern Ontario School of Medicine at Laurentian University: Northern Ontario School of Medicine East Campus
\end{abstract}

\section{Case report}

Keywords: Autoimmune progesterone dermatitis, bilateral oophorectomy, omalizumab

Posted Date: November 19th, 2020

DOI: https://doi.org/10.21203/rs.3.rs-108412/v1

License: (a) (1) This work is licensed under a Creative Commons Attribution 4.0 International License. Read Full License 


\section{Abstract}

Background Autoimmune progesterone dermatitis (APD) is a rare skin condition caused by sensitivity to high levels of progesterone secreted during the luteal phase of the menstrual cycle. This may be due to various pathophysiological mechanisms including a Type I and Type IV hypersensitivity reaction. Here we present the case of a patient with APD whose episodic flares were controlled by the addition of omalizumab, after a bilateral oophorectomy failed to resolve her symptoms.

Case Presentation A 34-year-old female presented to our Endocrine Clinic with marked Cushingoid features secondary to high-dose oral prednisone prescribed for APD diagnosed 6 years earlier. She first developed a pruritic maculopapular rash on her arms and legs just after the birth of her second child in 2009. The rash was also associated with headaches and diffuse angioedema. It presented in a cyclical fashion, beginning one to two weeks before the start of her menstrual cycle, and ending shortly after it was complete. The severity of symptoms increased as time went on, and flare-ups began to also include dyspnea, nausea, vomiting and abdominal pain. Her symptoms improved with administration of oral prednisone, but she continued to experience breakthrough symptoms. After multiple failed treatment modalities, she elected bilateral oophorectomy in 2018. However, her symptoms of APD persisted and she still required high-dose oral prednisone. Her condition was further complicated by vasomotor menopausal symptoms and progressive iatrogenic Cushing's syndrome. She eventually was started on Omalizumab, which suppressed further recurrences of APD symptoms and allowed her to wean off prednisone. Vasomotor menopausal symptoms responded well to the addition of conjugated estrogens with bazedoxifene. However, her symptoms of diffuse bony pain and arthralgias which started whilst on prednisone have persisted in spite of discontinuing prednisone.

Conclusions To our knowledge, this is only the third case of APD which was successfully treated with Omalizumab and the first case where a bilateral oophorectomy failed to resolve symptoms of APD in the literature. This case also demonstrates the complications of vasomotor menopausal symptoms secondary to a bilateral oophorectomy, as well as the adverse effects of long-term glucocorticoid therapy.

\section{Background}

Autoimmune progesterone dermatitis (APD) is a rare condition, with a literature review from 2016 documenting approximately 90 cases [1]. Patients with APD are sensitive to the high levels of progesterone secreted during the luteal phase of the menstrual cycle [2]. Depending on whether the reaction is IgE-mediated, or T-cell mediated, APD can present in various forms, such as eczema, folliculitis, and erythema multiforme, but can also progress to more acutely severe manifestations such as dyspnea and anaphylaxis [2]. Symptom onset is typically one week prior to menses and symptoms resolve a few days after the onset of menstruation [3,4]. 
Although the pathogenesis of the disease is not yet fully understood, it has been postulated to involve Type I and Type IV hypersensitivity reactions [4]. Progesterone has been noted to stimulate immunoglobulin $\mathrm{E}(\mathrm{lgE})$ - mast cell degranulation [2,5]. Additionally, progesterone sensitivity may be also due to cell-mediated immunity, due to prior uptake of progesterone by antigen-presenting cells and stimulation of T-helper cells [4]. Furthermore, cross-reactivity with other endogenous steroid hormones such as 17-a-hydroxy-progesterone may also be a plausible mechanism for increased progesterone sensitivity in APD patients [1].

The diagnosis of APD is based on the unique cyclical appearance of symptoms during a menstrual cycle. If APD is IgE-mediated, the diagnosis is confirmed by a positive wheal and flare response to skin testing with progesterone [6]. Various treatment options have been described for APD, including gonadotrophinreleasing hormone ( $\mathrm{GnRH}$ ) agonists (suppressing progesterone production), oral contraceptives, tamoxifen, prednisone, dapsone, thalidomide, azathioprine and danazol [7-9]. However, if these therapies fail, or cause intolerable side effects, a bilateral oophorectomy can be performed as a more durable solution [7].

\section{Case Presentation}

A 34-year-old female with pronounced Cushingoid features presented to our Endocrinology clinic in 2018. She had been taking high dose oral prednisone for a known diagnosis of severe autoimmune progesterone urticaria, refractory to bilateral oophorectomy.

Figure 1: Acute Angioedema- Patient Photograph, Consent Obtained

A raised pruritic macular erythematous rash initially appeared on her arms and legs in 2009 , two to three days after the birth of her second child. This rash was associated with headaches, and diffuse angioedema of her hands, feet, lips, and eyelids (Figure 1). The rash continued to occur in a cyclical fashion, beginning one to two days before the start of her menstrual cycle, and resolving a few days following the end of her cycle. With each subsequent menstrual cycle, the rash seemed to become more diffuse, and she developed dyspnea, most likely a result of laryngeal edema. Her flare-ups also coincided with nausea, dizziness, and abdominal cramping.

For several years, she was treated with numerous medications, including first and second generation $\mathrm{H} 1$ and $\mathrm{H} 2$ histamine antagonists, tricyclic antidepressants, calcineurin inhibitor immunosuppressant therapy and psoralen and ultraviolet A (PUVA) therapy. These treatments were all unsuccessful in improving her symptoms. She was eventually started on oral prednisone in doses of up to $100 \mathrm{mg}$ daily. Symptomatic improvement was achieved with corticosteroid use, but pre-menstrual flare-ups would still occur quite often. After three years of persistent symptoms, she was referred to an Immunologist who established a likely diagnosis of autoimmune progesterone dermatitis with potential catamenial anaphylaxis. This was confirmed using a progesterone skin test. 
She was then started on a trial of a low-dose oral contraceptive pill, which contained $0.02 \mathrm{mg}$ ethinyl estradiol and $0.1 \mathrm{mg}$ of levonorgestrel, in an attempt to be desensitized to progesterone. High dose prednisone was prescribed for flare-ups. This was an attempt to wean her off prednisone. After 6 months, there was still no improvement in her symptoms. Due to the long-term use of high-dose prednisone, she developed Cushingoid features including moon face, violaceous striations, alopecia, and central adiposity (Figure 2 - left panel).

Due to the progressive side effects of prednisone as well as persistent symptoms, she elected to undergo a bilateral oophorectomy at age 34 in June 2018. However, her flare-ups of urticaria with angioedema persisted and treatment with prednisone $50 \mathrm{mg}$ daily was reinstituted.

Unfortunately, following oophorectomy, her condition was further complicated by vasomotor menopausal symptoms, progressive iatrogenic Cushing's syndrome and unresolved APD.

\section{Discussion}

\section{Case Dilemma \#1: Refractory APD following Bilateral Oophorectomy}

In our case, the patient had elected to proceed with a bilateral oophorectomy after three years of unresolving symptoms despite various treatment modalities. Bilateral oophorectomy for unresolving APD has been used 19 times and was found to be successful in all reported cases [1,10-14]. However, in our case, the patient continued to have breakthrough episodes of generalized urticaria and peripheral swelling. These symptoms were in line with what she was experiencing before her bilateral oophorectomy. The differential diagnosis may also include chronic spontaneous urticaria and angioedema post-oophorectomy. Unfortunately, she has not been retested using a medroxyprogesterone skin test since her procedure. To our knowledge, this is the first case where a bilateral oophorectomy procedure may have failed to resolve symptoms of APD. However, one potential publication bias could indeed be a lack of reporting of non-responsive cases.

Three months following bilateral oophorectomy, she presented to our Endocrine clinic for an initial consultation for Cushing syndrome while being treated with $50 \mathrm{mg}$ of daily prednisone. In search of a prednisone sparing treatment for her APD, we initiated Omalizumab (Xolair) monoclonal antibody therapy. Cross-linking of IgE or stimulation of the lgE receptor on mast cells leads to release of mast cell mediators including histamine. Omalizumab works by decreasing the amount of free IgE antibodies in circulation leading to a downregulation of IgE receptors on mast cells, thereby inhibiting the release of mast cell mediators $[15,16]$. IgE facilitates stimulation of histamine, causing the immediate hypersensitivity reaction. Subcutaneous injections of Omalizumab $300 \mathrm{mg}$ allowed the patient to eliminate the requirement of daily oral prednisone over the course of six months with a slow taper. Most significantly, her ADP flare-ups have resolved. This is consistent with two other case reports which have shown improvement in symptoms of APD with Omalizumab $[17,18]$. This is the third case for which Omalizumab was used for treatment for APD to our knowledge. We continue to attempt Omalizumab dose and frequency reduction, which she has not tolerated so far. 
Case Dilemma \#2: Complications of Early Menopause Secondary to a Bilateral Oophorectomy Procedure

Following her bilateral oophorectomy as well as chronic prednisone use, our patient was at risk for symptomatic early menopause and premature osteoporosis. As she had not responded to progesterone desensitization in the past, typical hormone replacement therapy, consisting of estrogen and progesterone, could not be considered. In addition, her uterus remained in situ, therefore treatment with unopposed estrogen would have put her at risk for endometrial hyperplasia and subsequent malignancy [19]. In hindsight, consideration of hysterectomy in addition to the bilateral oophorectomy would have negated the requirement of progesterone and allow for estradiol monotherapy. A hysterectomy may still be considered for the future. Unfortunately, estradiol monotherapy was not possible for our patient. As a result, we started the patient on the combination of conjugated estrogens with bazedoxifene. Several large Phase 3 trials have shown this combination to be effective in reducing vasomotor menopausal symptoms, as well as maintaining bone health and reducing the risk of endometrial hyperplasia for postmenopausal women between 40-65 years of age with an intact uterus [20-22]. Initiation of this treatment resulted in immediate vasomotor symptom relief.

\section{Case Dilemma \#3: The Consequences of Long-Term Use of High Dose Oral Prednisone}

Our patient was on sporadic prednisone since 2008. Following her bilateral oophorectomy in June 2018, she was taking prednisone $50 \mathrm{mg}$ daily for three months prior to our initial consultation. Despite APD symptom improvement, long-term glucocorticoid usage has a myriad of possible adverse side effects, including lowered bone mass, myopathy, hyperglycemia, Cushing's syndrome, and iatrogenic adrenal insufficiency [23]. In fact, our patient suffered from most of these complications.

Figure 2: latrogenic Cushing's Syndrome (left panel - at the time of Xolair initiation), followed by drastic weight loss off prednisone (right panel - approximately one year steroid free), Patient Photograph, Consent Obtained

As expected, she developed significant Cushingoid features including a significant weight gain of 70lbs over four years, depression, proximal muscle wasting, moon face, easy bruising and dorsoclavicular adiposity. She has regained muscle mass and lost $35 \mathrm{lbs}$ since prednisone discontinuation with resolution of the Cushingoid features (Figure 2 - right panel).

Our patient's chronic use of glucocorticoid coupled with her bilateral oophorectomy puts her at significant risk for low bone mass and early development of osteoporosis. In August 2018, her bone mineral density showed lumbar spine osteopenia (T-Score of Lumbar Spine: -1.1, Z-Score of Lumbar Spine: -2.0, T-Score of Femoral Neck: -0.8, Z-Score of Femoral Neck: -1.1, T-Score of Total Hip: -0.5 and Z-Score of Total Hip: -0.9 ) and she was started on Calcium, Vitamin D supplements, as well as conjugated estrogens with bazedoxifene.

Furthermore, it was postulated that she had an episode of prednisone-induced adrenal insufficiency following a quick taper of daily oral prednisone, resulting in an episode of severe atypical chest pain, 
muscle aches, and nausea. Due to iatrogenic adrenal suppression, the steroid taper occurred very slowly over the course of six months. Since this time, the patient has remained off steroids and is asymptomatic with cortisol levels within physiological range.

Throughout the many years of glucocorticoid therapy, she began to develop diffuse indeterminate bone pain and arthralgias which persist to this day. Multiple imaging modalities were ordered, which revealed no bone abnormalities. Muscle and joint pain have been reported with conjugated estrogens with bazedoxifene [24], however transient discontinuation of conjugated estrogens with bazedoxifene did not improve symptoms. Omalizumab serum sickness and other possible rheumatological conditions were also ruled out by her Immunologist. The cause of the symptoms remains inconclusive, but they are improving with physiotherapy.

\section{Conclusions}

This patient case depicts a complex case of APD which began following a pregnancy. In contrast to the 19 previously documented cases, a bilateral oophorectomy did not lead to resolution of symptoms. This unique case identifies an additional steroid sparing therapeutic option, omalizumab, that can be considered for the treatment of APD. Given the apparent effectiveness of Omalizumab, consideration to using this drug should be given before proceeding on to bilateral oophorectomy surgery for APD.

This case also highlights the unique role of conjugated estrogens with bazedoxifene for vasomotor menopause treatment in patients with APD post bilateral oophorectomy if the uterus remains intact. Again, perhaps hysterectomy should be considered in future cases as it may allow estrogen monotherapy.

Lastly, this case report demonstrates the complications of long-term steroid therapy as well as the challenges encountered in discontinuing their use. Omalizumab therapy allowed for steroid discontinuation and the conjugated estrogens with bazedoxifene allowed for bone protection against steroid induced bone demineralization.

\section{Abbreviations}

APD: Autoimmune progesterone dermatitis; IgE: Immunoglobulin E; GnRH: Gonadotropin releasing hormone; PUVA: Psoralen and Ultraviolet A.

\section{Declarations}

\section{Acknowledgements}

None.

\section{Authors' Contributions}


AV drafted the manuscript. AA cared for the patient and was involved in revising the manuscript. Additionally, HK, PV, SVU were also involved in revising the manuscript. All authors have read and approved the final manuscript.

\section{Funding}

None.

\section{Availability of Data and Materials}

The data use and analyzed during the current study are available from the corresponding author on reasonable request.

Ethics approval and consent to participate

An informed consent form was obtained from the patient to use her data.

Consent for publication

Consent for publication was obtained from the patient.

Competing interests

HK has been on an advisory board and speaker's bureau for Novartis Canada. AV, TP, SVU, PV and AA have no competing interests to disclose.

\section{Author Details}

1. Division of Internal Medicine, Northern Ontario School of Medicine, Sudbury, ON, Canada Akshay Varghese, Alescia Azzola

2. Division of Endocrinology, Schulich School of Medicine and Dentistry, Western University, London, ON, Canada

Terri Paul, Stan Van Uum

3. Division of Clinical Immunology and Allergy, Schulich School of Medicine and Dentistry, Western University, London, ON, Canada

Harold Kim

4. Division of Clinical Immunology and Allergy, St. Michael's Hospital, University of Toronto, Toronto, ON, Canada

Peter Vadas

Corresponding Author

Akshay Varghese: avarghese@nosm.ca 
1. Division of Internal Medicine, Northern Ontario School of Medicine, Sudbury, ON, Canada

\section{References}

1. Nguyen T, Ahmed AR. Autoimmune progesterone dermatitis: update and insights. Autoimmunity reviews. 2016;15(2):191-7.

2. Mbonile L. Autoimmune progesterone dermatitis: Case report with history of urticaria, petechiae and palpable pinpoint purpura triggered by medical abortion. South African Medical Journal. 2016;106(4):356-8.

3. Snyder JL, Krishnaswamy G. Autoimmune progesterone dermatitis and its manifestation as anaphylaxis: a case report and literature review. Annals of Allergy, Asthma \& Immunology. 2003;90(5):469-77.

4. Prieto-Garcia A, Sloane DE, Gargiulo AR, Feldweg AM, Castells M. Autoimmune progesterone dermatitis: clinical presentation and management with progesterone desensitization for successful in vitro fertilization. Fertility and sterility. 2011;95(3):1121-e9.

5. Camões S, Sampaio J, Rocha J, Tiago P, Lopes C. Autoimmune progesterone dermatitis: Case report of an unexpected treatment reaction. Australasian Journal of Dermatology. 2017;58(3):e132-4.

6. Solomon M, Itsekson AM, Lev-Sagie A. Autoimmune progesterone dermatitis. Current Dermatology Reports. 2013;2(4):258-63.

7. Medeiros S, Rodrigues-Alves R, Costa M, Afonso A, Rodrigues A, Cardoso J. Autoimmune progesterone dermatitis: treatment with oophorectomy. Clinical and experimental dermatology. 2010;35(3):e12-3.

8. Zhang M, Tang $X$, Zhou H, Liao Q, Han J. Case of autoimmune progesterone dermatitis presenting as necrotic migratory erythema successfully controlled by danazol. The Journal of Dermatology. 2020;47(2):178-80.

9. Le K, Wood G. A case of autoimmune progesterone dermatitis diagnosed by progesterone pessary. Australasian journal of dermatology. 2011;52(2):139-41.

10. Whitt W, Stiegler JD, Richardson CT. Autoimmune progesterone dermatitis mimicking facial erythromelalgia successfully treated with hysterectomy and bilateral salpingo-oophorectomy. JAAD Case Reports. 2020;6(10):1104-6.

11. Galán-Gutierrez M, Gomez-Arias PJ, Rodenas-Herranz T, Ruiz-Villaverde R. Autoimmune progesterone dermatitis: Successful outcome with bilateral salpingo-oophorectomy. Dermatologic Therapy. 2020.

12. Drayer SM, Laufer LR, Farrell ME. Autoimmune progesterone dermatitis presenting as StevensJohnson syndrome. Obstetrics \& Gynecology. 2017;130(4):881-4.

13. DeRosa I, Bender B, Centilli M. Autoimmune progesterone dermatitis. Cutis. 2018;(4):E12-4.

14. Grunnet KM, Powell KS, Miller IA, Davis LS. Autoimmune progesterone dermatitis manifesting as mucosal erythema multiforme in the setting of HIV infection. JAAD Case Reports. 2017;3(1):22. 
15. Spector SL, Tan RA. Effect of omalizumab on patients with chronic urticaria. Annals of Allergy, Asthma \& Immunology. 2007;99(2):190-3.

16. Chang TW, Chen C, Lin CJ, Metz M, Church MK, Maurer M. The potential pharmacologic mechanisms of omalizumab in patients with chronic spontaneous urticaria. Journal of Allergy and Clinical Immunology. 2015;135(2):337-42.

17. Heffler E, Fichera S, Nicolosi G, Crimi N. Anaphylaxis due to progesterone hypersensitivity successfully treated with omalizumab.

18. Gadoury-Levesque V, Bernstein J. A CASE OF REFRACTORY RECURRENT CYCLICAL ANGIOEDEMA AND URTICARIA SUCCESSFULLY TREATED WITH MULTIPLE STEPS THERAPY. Annals of Allergy, Asthma \& Immunology. 2018 Nov 1;121(5):S85.

19. Gompel A. Progesterone and endometrial cancer. Best Practice \& Research Clinical Obstetrics \& Gynaecology. 2020 Jun 9.

20. Pinkerton JV, Utian WH, Constantine GD, Olivier S, Pickar JH. Relief of vasomotor symptoms with the tissue-selective estrogen complex containing bazedoxifene/conjugated estrogens: a randomized, controlled trial. Menopause. 2009;16(6):1116-24.

21. Pinkerton JV, Harvey JA, Lindsay R, Pan K, Chines AA, Mirkin S, Archer DF, SMART-5 Investigators. Effects of bazedoxifene/conjugated estrogens on the endometrium and bone: a randomized trial. The Journal of Clinical Endocrinology \& Metabolism. 2014;99(2):E189-98.

22. Silverman SL, Christiansen C, Genant HK, Vukicevic S, Zanchetta JR, de Villiers TJ, Constantine GD, Chines AA. Efficacy of bazedoxifene in reducing new vertebral fracture risk in postmenopausal women with osteoporosis: results from a 3-year, randomized, placebo-, and active-controlled clinical trial. Journal of bone and mineral research. 2008;23(12):1923-34.

23. Oray M, Abu Samra K, Ebrahimiadib N, Meese H, Foster CS. Long-term side effects of glucocorticoids. Expert opinion on drug safety. 2016;15(4):457-65.

24. Chaplin S. Duavive HRT: conjugated oestrogens with bazedoxifene. Prescriber. 2016;(12):53-.

\section{Figures}




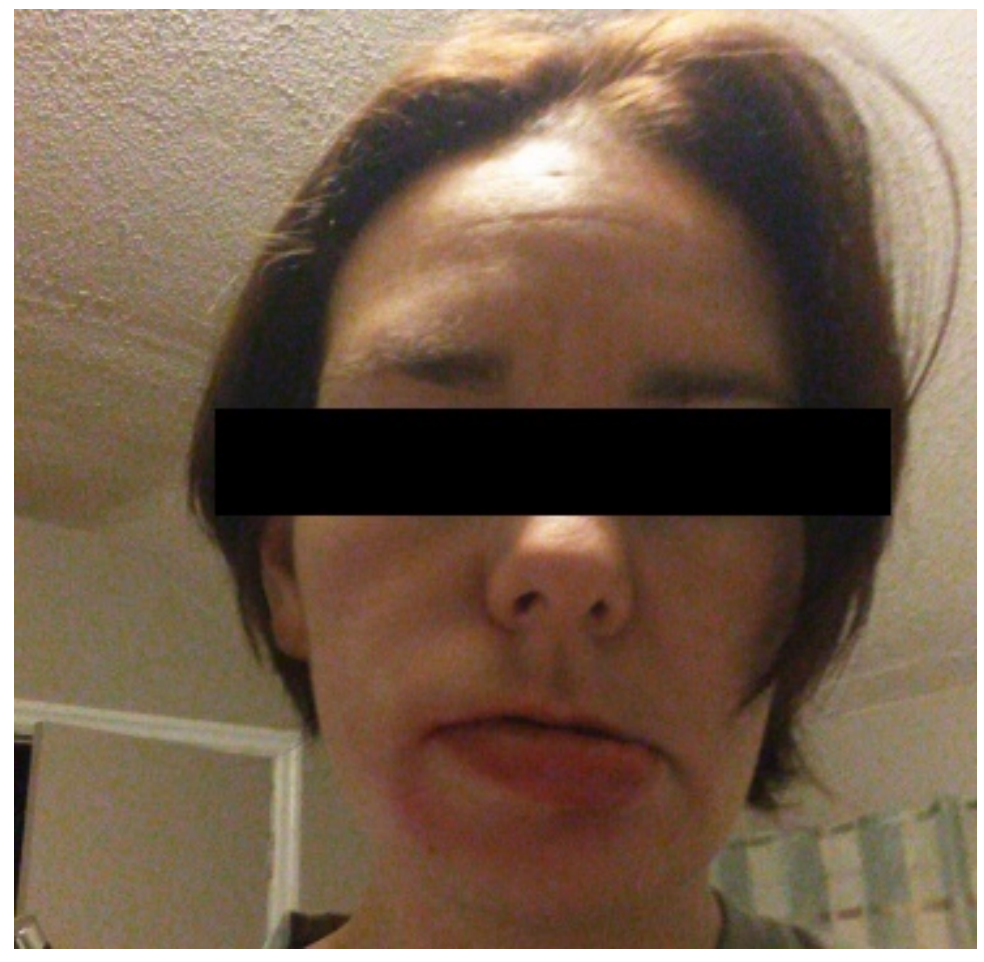

\section{Figure 1}

Acute Angioedema- Patient Photograph, Consent Obtained

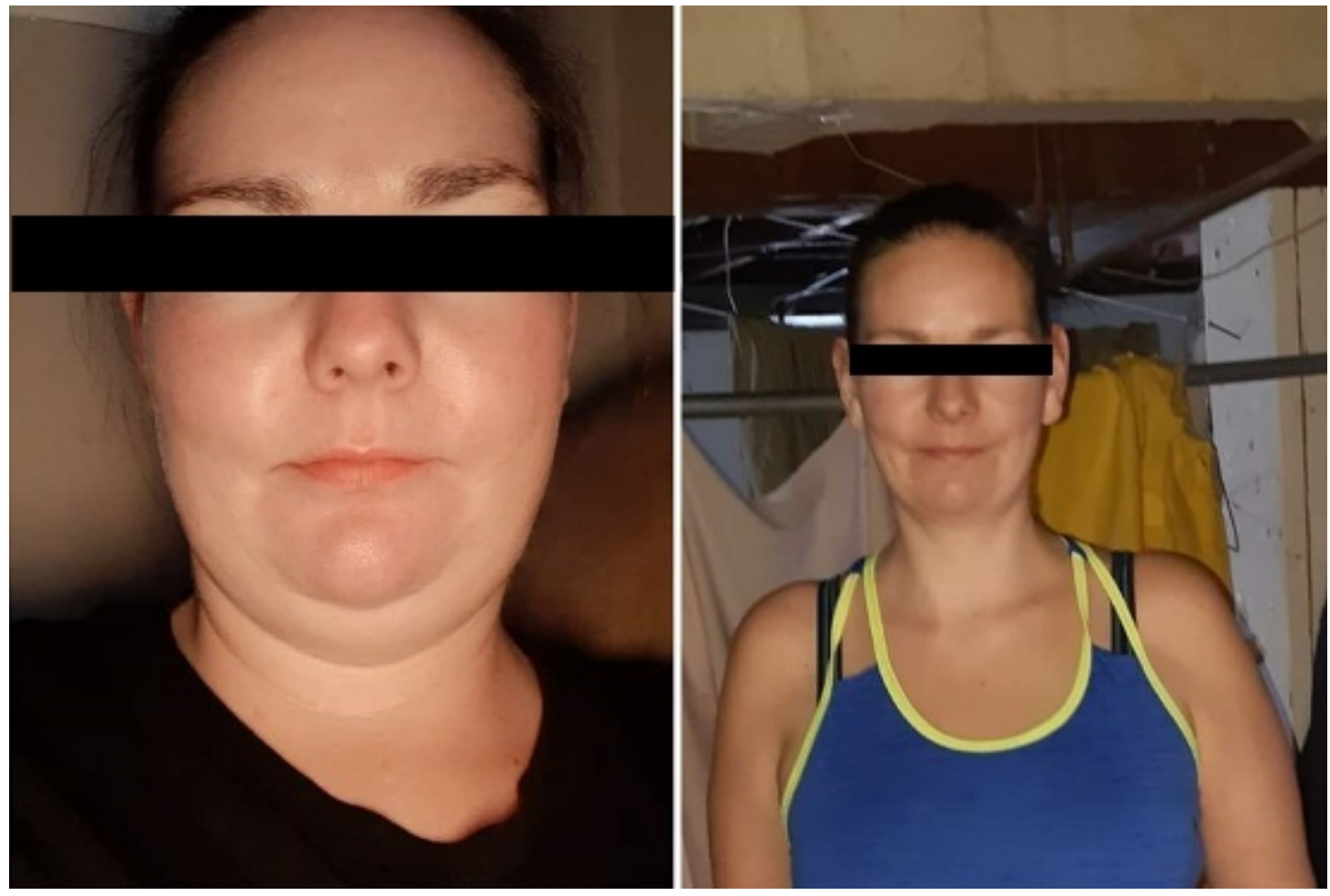

Figure 2

latrogenic Cushing's Syndrome (left panel - at the time of Xolair initiation), followed by drastic weight loss off prednisone (right panel - approximately one year steroid free), Patient Photograph, Consent Obtained 